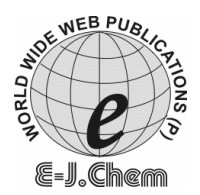

http://www.e-journals.net
ISSN: 0973-4945; CODEN ECJHAO

E-Journal of Chemistry

Vol. 5, No.3, pp. 584-592, July 2008

\title{
Tetrahydroxy Cyclic Urea-Potent Inhibitor for HIV-1 Protease Wild Type and Mutant Type - A Computational Design
}

\author{
S. SREE KANTH and M. VIJJULATHA* \\ Department of Chemistry, Nizam College, Osmania University, \\ Basheer Bagh, Hyderabad- 500 001, India \\ drmvl2002@yahoo.com
}

Received 7 November 2007; Accepted 5 January 2008

\begin{abstract}
A series of novel tetrahydroxy cyclic urea molecules as HIV-1 protease inhibitors were designed using computational techniques. The designed molecules were compared with the known cyclic urea molecules by performing docking studies on six of wild type protein and three mutant protein varieties and calculating their ADME properties. A series of novel molecules were designed by substituting hydrogen at the $\mathrm{P}_{1} / \mathrm{P}_{1}{ }^{\prime}$ positions with hydroxyl group increasing the bioavailability these had better ADME properties and binding affinity towards HIV-1 protease. The biological activity of these inhibitors were predicted by a model equation generated by the regression analysis between biological activity $(\log 1 / \mathrm{Ki})$ of known inhibitors and there combined docking scores from six of the wild type protein docking. The synthetic studies are in progress.
\end{abstract}

Keywords: Docking, ADME, OPLS-AA, MMFF94s, CU (Cyclic urea), THCU (Terahydroxy cyclic urea).

\section{Introduction}

AIDS is one of the most serious health problems today. Human immune virus type 1 (HIV-1) is the main causative agent of AIDS. Inhibition of virally encoded protease has been demonstrated to be an effective antiviral drug therapy against HIV-1 infection ${ }^{1}$ Several HIV-1 protease inhibitors have been shown to reduce the viral load and increase the number of $\mathrm{CD} 4^{+}$ lymphocytes in HIV infected patients ${ }^{2}$.

HIV protease inhibitors belong to three different classes as reported in the literature, nonhydrolysable transition state isosteres, similar to renin inhibitors; pseudo symmetrical and C-2 symmetrical compounds ${ }^{3}$ and nonpeptidic inhibitors ${ }^{4}$. Cyclic ureas have shown good HIV protease inhibition. The cyclic urea classes of inhibitors are designed to displace a unique structural water molecule present in the HIV-1 protease active site ${ }^{5}$. These inhibitors have 
the hydroxyl groups and carbonyl oxygen positioned which is an essential factor for high potency. The diols i.e, the hydroxyl groups interact with ASP25/25' and the carbonyl oxygen with ILE50/50'. Amino acid residues like ASP30/30', GLY48/48' and ARG8/8' present in the active site also play an important role in inhibitor binding.

\section{Experimental}

\section{Method and software}

The computational work was run on a $3.2 \mathrm{GHz}$ Intel Pentium-4 systems. The software ${ }^{6}$ Glide 4.0 was used for protein minimization, grid generation and ligand docking Qikprop 2.5 was used $^{7}$ to calculate the ADME properties of the ligands

Nine crystal structures of HIV-1 protease were downloaded form protein data bank out of which three were mutant variety ( Wild type pdb id: 1PRO, 1BVG, 1AJX, 1SBG, 1HSG and 2UPJ. Mutant type pdb $i d$ : $1 \mathrm{MER}, 1 \mathrm{MEU}$ and $1 \mathrm{MET})^{8-14}$. These protein structures were prepared by glide application's protein preparation job. The protein preparation facility consists of two components, preparation and refinement. After ensuring chemical correctness, the preparation component adds hydrogen and neutralizes side chains that are not close to the binding cavity and do not participate in salt bridges. The refinement component performs a restrained impact minimization of the co-crystallized complex, which reorients side-chain hydroxyl groups and alleviates potential steric clashes. It uses the OPLS-AA force field for this purpose and then the active site of the protein was defined and a grid was prepared for each of the protein structure with receptor Vander Waals scaling for the non polar atoms as 0.9 (which makes the protein site "roomier" by moving back the surface of non-polar regions of the protein and ligand. This kind of adjustments emulate to some extent the effect of breathing motions to the protein site it is a kind of giving breathing to the receptor). The ligands were built using maestro build panel and prepared by Lig Prep2.0 application. LigPrep produces the low energy conformer of the ligand using the MMFF94s force field. The ADME properties (required pharmacokinetic properties of viable drug compounds) were calculated by Qikprop 2.5. The lower energy conformations of the ligands were selected and were docked into the grid generated from the nine protein structures using the standard precision docking mode ${ }^{15}$. In this docking method the ligands are flexible and the receptor is rigid, except the protein active site has slight flexibility. To include receptor flexibility the ligands were docked into different grid generated for nine protein conformations ${ }^{16,17}$. The known inhibitors which docked into the six of the wild type protein were selected and a regression analysis was performed by taking the combined docking scores of each ligand. The combined docking scores were calculated by taking the sum of glide score (docking scores) of each ligand form the six docking processes.

\section{Results and Discussion}

ADME properties of cyclic urea chosen from literature ${ }^{18-24}$ with varied range of inhibition constant were calculated (structures shown in Figure 1). The calculated ADME properties showed poor bioavailability. The required pharmacokinetic properties for a viable drug compound are ${ }^{25}$.

(i) $150 \leq \mathrm{MW} \leq 650$

(ii) $-1 \leq \log \mathrm{Po} / \mathrm{w} \leq 6.5$

(iii) $\log \mathrm{S} \geq-7$

(iv) Pmdck $\geq 5$

(v) $\log \mathrm{BB} \geq-3$ 
The first three are based on Lipinski rule of five ${ }^{26,27}$, molecular weight less than 650 , partition coefficient between octanol and water $(\log \mathrm{Po} / \mathrm{w})$ between -1 and 6.5 and solubility $(\log S)$ greater than -7 . Pmdck and Log BB parameter tells about the ability of the drug to pass through blood brain barrier which is mandatory for inhibition of HIV infection. The ADME values of known inhibitors are given in Table 1.<smiles>[2H]N1C(=O)N(P)[C@H]([PH])[C@@H](O)[C@H](O)[C@H]1P</smiles>

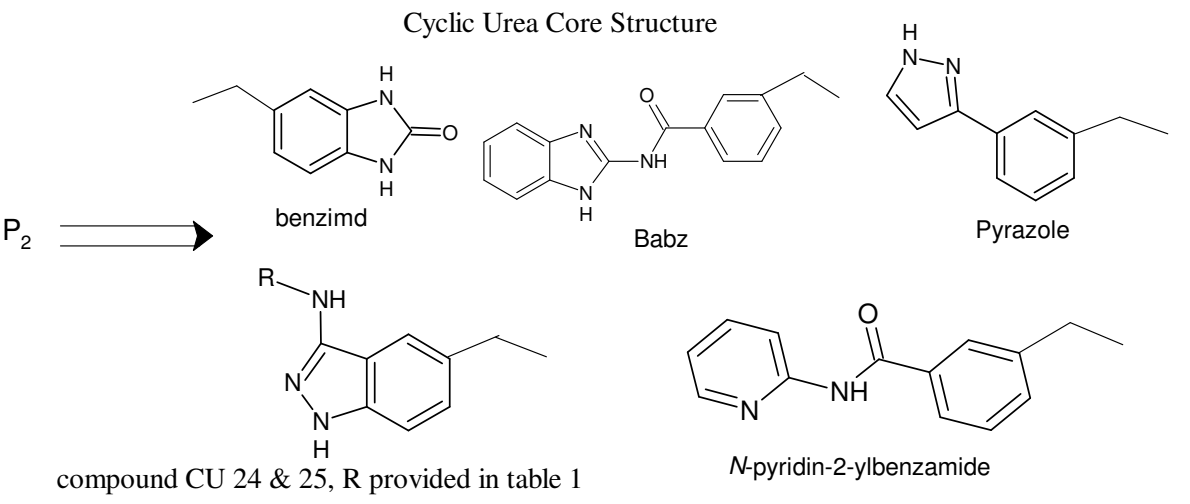

Figure 1. Structure of Cyclic urea inhibitors. $P_{2}$ and $P_{1}$ substitutions provided in Table 1 .

Table 1. ADME properties of cyclic urea inhibitors

\begin{tabular}{|c|c|c|c|c|c|c|c|}
\hline $\begin{array}{l}\text { Compd. } \\
\text { No. }\end{array}$ & $\mathrm{P} 2$ & $\mathrm{P} 1$ & $\begin{array}{c}\text { M. } \\
\text { weight }\end{array}$ & $\begin{array}{l}\mathrm{Log} \\
\mathrm{Po} / \mathrm{W}\end{array}$ & $\log S$ & $\log B B$ & Pmdck \\
\hline CU 1 & $n$-propyl & Benzyl & 410.55 & 5.560 & -6.020 & -0.549 & 2174.33 \\
\hline CU 2 & $n$-butyl & Benzyl & 438.60 & 6.341 & -6.898 & -1.056 & 2172.22 \\
\hline CU 3 & $n$-hexyl & Benzyl & 494.71 & 7.919 & -8.624 & -1.454 & 2172.65 \\
\hline CU 4 & $i$-butyl & Benzyl & 438.60 & 6.134 & -6.275 & -0.739 & 2785.55 \\
\hline CU 5 & $i$-pentyl & Benzyl & 466.66 & 6.957 & -7.536 & -1.058 & 2183.61 \\
\hline CU 6 & $i$-hexyl & Benzyl & 494.71 & 7.804 & -8.618 & -1.265 & 2236.78 \\
\hline CU 7 & $i$-heptyl & Benzyl & 522.76 & 8.603 & -9.439 & -1.472 & 2172.64 \\
\hline CU 8 & Neo-hexyl & Benzyl & 494.71 & 7.579 & -8.009 & -0.997 & 2488.23 \\
\hline CU 9 & Iso prenyl & Benzyl & 430.54 & 5.815 & -5.989 & -0.782 & 2500.15 \\
\hline CU 10 & $\begin{array}{l}\text { Cyclo propyl } \\
\text { methyl }\end{array}$ & Benzyl & 434.57 & 5.708 & -5.839 & -0.911 & 1637.57 \\
\hline CU 11 & $\begin{array}{l}\text { Cyclo butyl } \\
\text { methyl }\end{array}$ & Benzyl & 462.63 & 6.245 & -6.319 & -0.882 & 1769.91 \\
\hline CU 12 & $\begin{array}{l}\text { Cyclo pentyl } \\
\text { methyl }\end{array}$ & Benzyl & 490.68 & 6.801 & -6.741 & -0.825 & 2043.12 \\
\hline CU 13 & $m$ - fluro benzyl & Benzyl & 542.62 & 7.379 & -7.508 & -0.776 & 2556.25 \\
\hline
\end{tabular}




\begin{tabular}{|c|c|c|c|c|c|c|c|}
\hline CU 14 & $O$ - fluro benzyl & Benzyl & 542.62 & 7.328 & -7.231 & -0.749 & 2686.60 \\
\hline CU 15 & $p$ - fluro benzyl & Benzyl & 542.62 & 7.492 & -7.693 & -0.776 & 2591.58 \\
\hline CU 16 & $\begin{array}{c}m \text { - chloro } \\
\text { benzyl }\end{array}$ & Benzyl & 575.53 & 7.895 & -8.069 & -0.777 & 2544.90 \\
\hline CU 17 & $p$ - chloro benzyl & Benzyl & 575.53 & 7.987 & -8.476 & 0.795 & 2581.98 \\
\hline CU 18 & $\begin{array}{c}m \text { - bromo } \\
\text { benzyl }\end{array}$ & Benzyl & 664.43 & 7.890 & -7.676 & -0.717 & 2751.75 \\
\hline CU 19 & $\begin{array}{l}m \text {-methyl } \\
\text { benzyl }\end{array}$ & Benzyl & 534.69 & 7.565 & -7.158 & -0.718 & 2757.74 \\
\hline CU 20 & $\begin{array}{c}m \text {-(tri fluro } \\
\text { methyl) benzyl }\end{array}$ & Benzyl & 642.63 & 8.446 & -8.517 & -0.754 & 2617.90 \\
\hline CU21 & $\begin{array}{c}p \text {-(tri fluro } \\
\text { methyl) benzyl }\end{array}$ & Benzyl & 642.63 & 9.039 & -10.014 & -0.756 & 3092.61 \\
\hline CU 22 & $\begin{array}{c}m \text {-(hydroxyl } \\
\text { methyl benzyl) }\end{array}$ & Benzyl & 566.69 & 5.511 & -5.376 & -1.756 & 362.72 \\
\hline CU 23 & benzimd & Benzyl & 618.69 & 4.426 & -6.614 & -3.142 & 5.428 \\
\hline CU 24 & $\begin{array}{c}\text { methyl } \\
\text { cyclopropyl }\end{array}$ & Benzyl & 724.90 & 7.316 & -6.956 & -2.439 & 100.72 \\
\hline CU 25 & $n$-butyl & Benzyl & 728.93 & 7.815 & -9.150 & -3.228 & 60.95 \\
\hline CU 26 & & Ben & & 8.476 & -10.706 & & 66 \\
\hline CU 27 & $\mathrm{Babz}$ & i-butyl & 756.90 & 7.490 & -9.883 & -3.237 & .08 \\
\hline CU 28 & Babz & Cyclo hexyl & 808.97 & 8.117 & -10.45 & -2.468 & 68.49 \\
\hline CU 29 & Babz & $p$-hydoxy benzyl & 856.93 & 7.093 & -9.389 & -4.329 & 4.02 \\
\hline CU30 & Babz & $p$-methoxy benzyl & 884.98 & 8.530 & -10.11 & -3.309 & 35.77 \\
\hline CU 31 & Benzyl & $p$-hydroxy benzyl & 538.64 & 5.671 & -6.282 & 2.019 & 147.55 \\
\hline CU 32 & Benzyl & $p$ - mehtoxy benzyl & 566.69 & 7.382 & -7.557 & 0.900 & 3095.42 \\
\hline CU 33 & Benzyl & $\begin{array}{l}\text { 4-2-hydroxyethoxy } \\
\text { benzyl }\end{array}$ & 626.74 & 6.135 & -7.269 & -2.760 & 18.475 \\
\hline CU 34 & Benzyl & $\begin{array}{l}\text { 4-(pyridin-4- } \\
\text { ylmethoxy) benzyl }\end{array}$ & 720.86 & 9.086 & -10.386 & -2.120 & 598.66 \\
\hline CU 35 & Benzyl & $\begin{array}{l}\text { 4-(2-morpholin-4- } \\
\text { ylethoxy)benzyl }\end{array}$ & 764.95 & 6.853 & -6.682 & -0.604 & 46.45 \\
\hline CU 36 & Pyrazole & $\begin{array}{l}\text { 4-(pyridin-4- } \\
\text { ylmethoxy) benzyl }\end{array}$ & 852.99 & 8.551 & -10.77 & -3.669 & 23.606 \\
\hline CU 37 & Pyrazole & $\begin{array}{l}\text { 4-[2-(dimethylamino) } \\
\text { ethoxy] benzyl }\end{array}$ & 813.00 & 6.826 & -7.55 & -2.197 & 1.848 \\
\hline CU 38 & $p$-Nitro benzyl & Benzyl & 596.63 & 6.028 & -7.178 & -2.805 & 21.937 \\
\hline CU 39 & $p$-Amino benzyl & & 536.67 & 5.322 & -6.217 & -2.318 & 120.619 \\
\hline CU 40 & $m$-Cyano benzyl & Benzyl & 556.66 & 5.670 & -7.401 & -2.314 & 64.322 \\
\hline CU 41 & $\begin{array}{l}N \text {-pyridin-2-yl- } \\
\text { benzamide }\end{array}$ & Benzyl & 746.86 & 7.337 & -9.467 & -2.795 & 91.815 \\
\hline
\end{tabular}

The compound $\mathrm{CU} 23$ the best molecule in the series with $\mathrm{Ki}<0.01 \mathrm{nM}$ had Pmdck value (5.428) and Log BB value (-3.192) less than required. The best docked compound from the series CU 37, CU 29 and CU 26 with combined docking scores -66.81, -66.74 and -66.2 respectively (Combined dock scores are provided in Table 2) which had good biological activity (Ki in nM range) had high molecular weight, their Log Po/w (6.826, 7.093 and 8.476 respectively) and Log S (-7.55, 9.389 and -10.706 respectively) value were not in the acceptable range. The Pmdck value for CU 37 and CU 29 were also very low, lesser than the acceptable range (1.848 and 4.02). The inhibitors CU 30, CU41, and CU 36 which have good biological activity were also having the lesser bioavailability. 
Table 2. Biological activity, combined dock score and predicted activity of known cyclic urea inhibitors.

\begin{tabular}{|c|c|c|c|c|c|}
\hline $\begin{array}{l}\text { Compd. } \\
\text { No. }\end{array}$ & $\mathrm{Ki}$ in $\mathrm{nM}^{*}$ & $\begin{array}{l}\text { Biological activity } \\
\quad(\log 1 / \mathrm{ki})\end{array}$ & $\begin{array}{l}\text { Combined dock score } \\
\text { (CDS) wile type }\end{array}$ & $\begin{array}{l}\text { Predicted activity } \\
\text { from Eq } 2\end{array}$ & $\begin{array}{c}\text { Combined } \\
\text { dock score } \\
(\mathrm{CDS}) \text { mutant }\end{array}$ \\
\hline $\mathrm{Cu} 1$ & 8 & 8.09691 & -45.49 & 8.332765 & -23.09 \\
\hline $\mathrm{Cu} 2$ & 1.4 & 8.853872 & -54.85 & 9.195289 & -27.8 \\
\hline $\mathrm{Cu} 3$ & 4.6 & 8.337242 & -50.87 & 8.828532 & -23.74 \\
\hline $\mathrm{Cu} 4$ & 49 & 7.309804 & -35.52 & 7.414029 & -20.32 \\
\hline $\mathrm{Cu} 5$ & 12 & 7.920819 & -52.38 & 8.967678 & -28.94 \\
\hline $\mathrm{Cu} 6$ & 7 & 8.154902 & -54.29 & 9.143685 & -24.78 \\
\hline $\mathrm{Cu} 7$ & 30 & 7.522879 & -45.04 & 8.291297 & -26.76 \\
\hline $\mathrm{Cu} 8$ & 36 & 7.443697 & -35.72 & 7.432459 & -22.83 \\
\hline $\mathrm{Cu} 9$ & 1.8 & 8.744727 & -47.59 & 8.52628 & -21.4 \\
\hline $\mathrm{Cu} 10$ & 2.1 & 8.677781 & -43.94 & 8.189932 & -25.39 \\
\hline $\mathrm{Cu} 11$ & 1.3 & 8.886057 & -54.5 & 9.163036 & -28.29 \\
\hline $\mathrm{Cu} 12$ & 4.3 & 8.366532 & -41.79 & 7.99181 & -16.77 \\
\hline $\mathrm{Cu} 13$ & 3 & 8.522879 & -49.33 & 8.686621 & -31.21 \\
\hline $\mathrm{Cu} 14$ & 34 & 7.468521 & -40.63 & 7.884916 & -23.78 \\
\hline $\mathrm{Cu} 15$ & 1.4 & 8.853872 & -42.2 & 8.029591 & -27.42 \\
\hline $\mathrm{Cu} 16$ & 0.89 & 9.05061 & -53.4 & 9.061671 & -33.1 \\
\hline $\mathrm{Cu} 17$ & 5.2 & 8.283997 & -41.23 & 7.940206 & -30.38 \\
\hline $\mathrm{Cu} 18$ & 1.4 & 8.853872 & -51.08 & 8.847883 & -31.11 \\
\hline $\mathrm{Cu} 19$ & 7 & 8.154902 & -45.35 & 8.319864 & -29.43 \\
\hline $\mathrm{Cu} 20$ & 22 & 7.657577 & -39.91 & 7.818568 & -27.55 \\
\hline $\mathrm{Cu} 21$ & 51 & 7.29243 & -43.9 & 8.186246 & -26.84 \\
\hline $\mathrm{Cu} 22$ & 0.14 & 9.853872 & -65.44 & 10.17116 & -36.67 \\
\hline $\mathrm{Cu} 23$ & 0.01 & 11 & -52.65 & 8.992559 & -30.71 \\
\hline $\mathrm{Cu} 24$ & 0.34 & 9.468521 & -49.42 & 8.694914 & -17.56 \\
\hline $\mathrm{Cu} 25$ & 1.3 & 8.886057 & -42.47 & 8.054472 & -8.45 \\
\hline $\mathrm{Cu} 26$ & 0.024 & 10.61979 & -66.2 & 10.24119 & -25.07 \\
\hline $\mathrm{Cu} 27$ & 0.3 & 9.522879 & -59.48 & 9.621943 & -25.68 \\
\hline $\mathrm{Cu} 28$ & 0.29 & 9.537602 & -57.54 & 9.443172 & -24.99 \\
\hline $\mathrm{Cu} 29$ & 0.035 & 10.45593 & -66.74 & 10.29095 & -17.18 \\
\hline $\mathrm{Cu} 30$ & 0.026 & 10.58503 & -65.94 & 10.21723 & -21.05 \\
\hline $\mathrm{Cu} 31$ & 1.1 & 8.958607 & -43.95 & 8.190854 & -8.79 \\
\hline $\mathrm{Cu} 32$ & 8.6 & 8.065502 & -44.42 & 8.234164 & -29.9 \\
\hline $\mathrm{Cu} 33$ & 0.76 & 9.119186 & -51.14 & 8.853412 & -25.18 \\
\hline $\mathrm{Cu} 34$ & 0.84 & 9.075721 & -50.34 & 8.779692 & -25.15 \\
\hline $\mathrm{Cu} 35$ & 5.9 & 8.229148 & -50.28 & 8.774163 & -9.17 \\
\hline $\mathrm{Cu} 36$ & 0.071 & 10.14874 & -55.14 & 9.222012 & -14.48 \\
\hline $\mathrm{Cu} 37$ & 0.061 & 10.21467 & -66.81 & 10.2974 & -5.14 \\
\hline $\mathrm{Cu} 38$ & 2.8 & 8.552842 & -49.41 & 8.693993 & -28.16 \\
\hline $\mathrm{Cu} 39$ & 1.1 & 8.958607 & -50.15 & 8.762184 & -28.47 \\
\hline $\mathrm{Cu} 40$ & 3 & 8.522879 & -52.95 & 9.020204 & -35.57 \\
\hline $\mathrm{Cu} 41$ & 0.043 & 10.36653 & -61.31 & 9.790578 & -30.55 \\
\hline
\end{tabular}

* Ki values from literature ref $18-24$ 
The new molecules (Terahydroxy's) were designed with different substitutions at the P2/P2' positions and substituting the hydrogen at $\mathrm{P} 1 / \mathrm{P}^{\prime}$ positions with hydroxyl group in order to increase the bioavailability (Structures shown in Figure 2). These molecules were first screened for their drug like properties (ADME are provided in Table 3). These had good bioavailability but because of larger no. of hydroxyl group they showed lesser Pmdck value but were in the acceptable range.

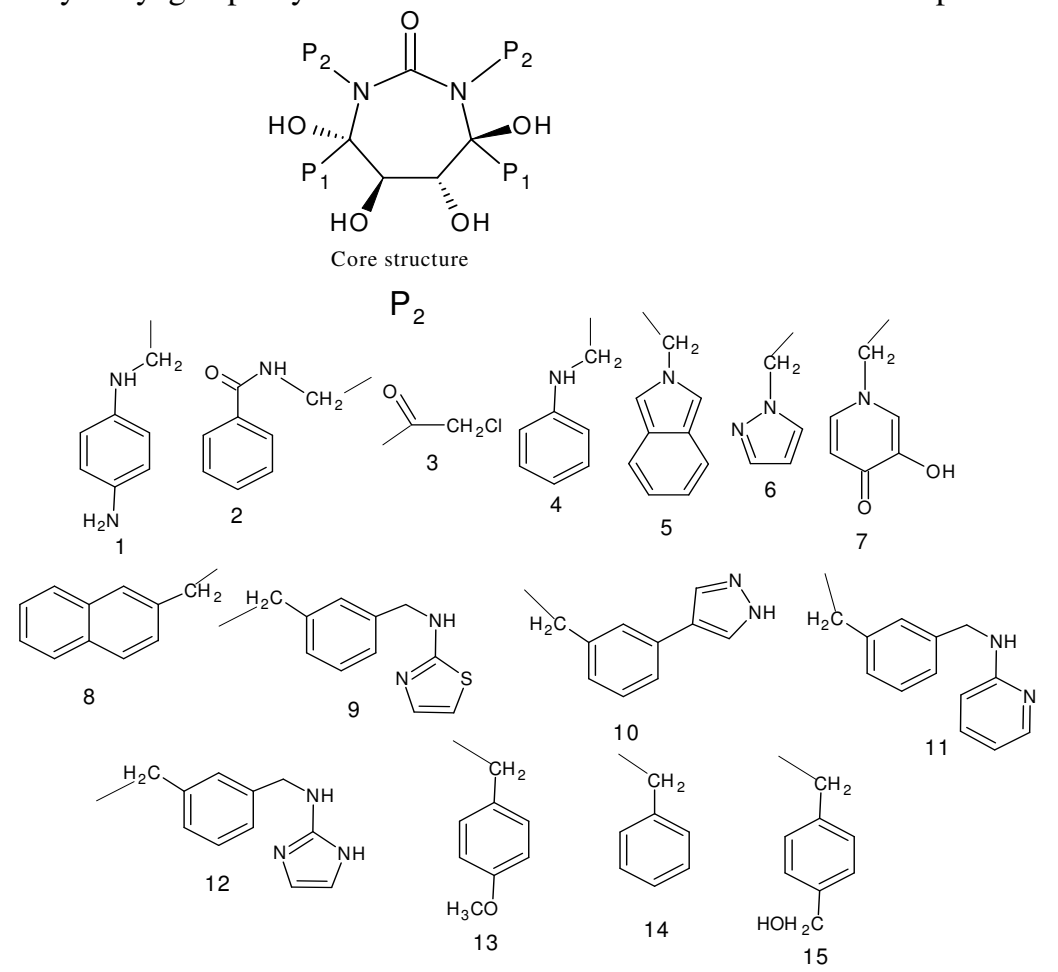

Figure 2. Structures of Terahydroxy molecules

Table 3. ADME properties of tetrahydroxy molecules

\begin{tabular}{|c|c|c|c|c|c|c|c|}
\hline Compd. No. & $\mathrm{P} 2$ & P1 & M. weight & Log Po/w & $\log S$ & $\log B B$ & Pmdck \\
\hline $\mathrm{OHC1}$ & 1 & Benzyl & 598.70 & 5.012 & -6.499 & -3.127 & 44.583 \\
\hline 2 & 2 & Benzyl & 624.69 & 5.927 & -6.789 & -1.926 & 398.31 \\
\hline 3 & 3 & Benzyl & 511.35 & 4.776 & -5.734 & -1.124 & 537.739 \\
\hline 4 & 4 & $p$ - Hydroxy benzyl & 600.67 & 5.446 & -6.712 & -2.934 & 67.402 \\
\hline 5 & 5 & $p$ - Hydroxy benzyl & 648.71 & 5.583 & -6.441 & -2.365 & 114.22 \\
\hline 6 & 6 & $p$ - Hydroxy benzyl & 550.57 & 2.239 & -3.605 & -2.603 & 36.672 \\
\hline 7 & 7 & Ethyl & 480.47 & 0.048 & -2.611 & -2.965 & 6.10 \\
\hline 8 & 8 & Ethyl & 514.62 & 5.412 & -5.913 & -1.062 & 1139.62 \\
\hline 9 & 9 & Ethyl & 638.79 & 4.801 & -5.342 & -1.971 & 394.59 \\
\hline 10 & 10 & Ethyl & 546.62 & 2.897 & -4.621 & -2.200 & 41.237 \\
\hline 11 & 11 & Ethyl & 626.75 & 5.848 & -6.226 & -1.868 & 623.77 \\
\hline 12 & 12 & Ethyl & 604.71 & 4.090 & -5.199 & -2.294 & 226.19 \\
\hline 13 & 13 & $p$-fluro phenyl & 510.53 & 4.192 & -4.753 & -1.191 & 1149.09 \\
\hline 14 & 14 & Fluro ethyl & 450.48 & 3.826 & -3.936 & -1.025 & 886.761 \\
\hline 15 & 15 & Fluro ethyl & 510.53 & 2.574 & -3.386 & -2.089 & 107.767 \\
\hline
\end{tabular}


The screened molecules were docked into the nine protein grids. These showed good docking scores compared to known inhibitors. Compound OHC-1, 2, and 4 with combined dock scores $-69.87,-63.45$, and -68.63 , respectively (Table 4) have ADME properties in acceptable range except for compound $\mathrm{OHC}-1$ which has less Log BB value than required, it may be due to presence of amine groups which restricts it from crossing the blood brain barrier.

Table 4. Combined dock score and predicted activity of novel tetrahydroxy molecules

\begin{tabular}{cccc}
\hline $\begin{array}{c}\text { Compd. } \\
\text { No. }\end{array}$ & $\begin{array}{c}\text { Combined dock score } \\
\text { wild type }\end{array}$ & $\begin{array}{c}\text { Combined dock score } \\
\text { mutant type }\end{array}$ & Predicted activity from Eq 2 \\
\hline OHC 1 & -69.87 & -34.96 & 10.57938 \\
2 & -63.45 & -29.14 & 9.987779 \\
3 & -55.24 & -30.82 & 9.231227 \\
4 & -68.63 & -42.76 & 10.46512 \\
5 & -48.74 & -36.98 & 8.632252 \\
6 & -43.35 & -24.31 & 8.135564 \\
7 & -36.77 & -29.91 & 7.529217 \\
8 & -49.19 & -31.08 & 8.67372 \\
9 & -49.71 & -37.98 & 8.721638 \\
10 & -47.48 & -26.45 & 8.516143 \\
11 & -56.22 & -32.85 & 9.321534 \\
12 & -57.69 & -38.79 & 9.456995 \\
13 & -37.33 & -25.35 & 7.580821 \\
14 & -37.14 & -23.56 & 7.563312 \\
15 & -40.2 & -23.56 & 7.845291 \\
\hline
\end{tabular}

A regression analysis of biological activity (Log1/Ki) and combined dock score for known inhibitor was carried out. The correlation coefficient $r=0.801$ and standard error of the estimate $\mathrm{s}=0.59$, and the following equation is found.

$$
\text { Biological activity }(\log 1 / \mathrm{Ki})=-0.09369 * \mathrm{cds}+4.111861
$$

The inhibitor CU 23 having $\mathrm{Ki}<0.01$ was taken as an outlier and the regression gave a correlation coefficient $r=0.8415$ and the standard error of estimate s reduced to 0.506 and following equation was obtained, the scatter plot is shown in Figure 3.

Biological activity $(\log 1 / \mathrm{Ki})=-0.09215^{*} \mathrm{cds}+4.140861$

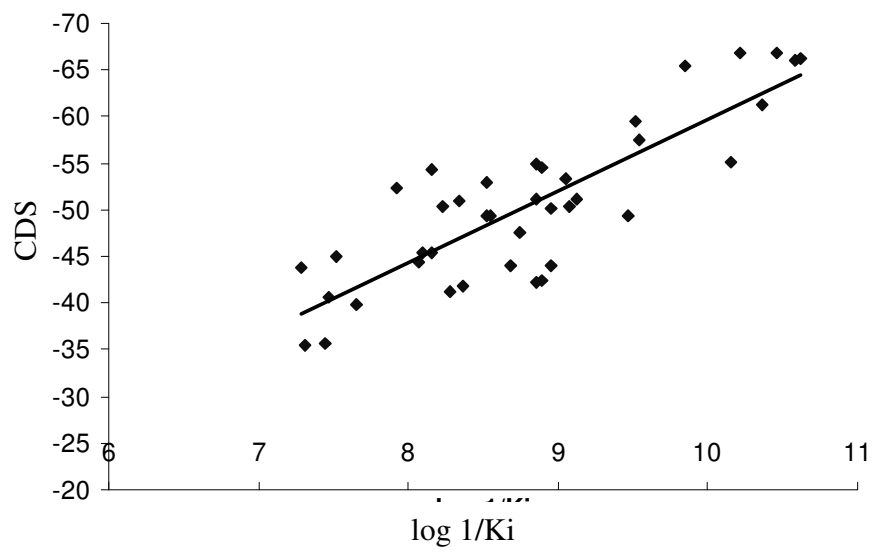

Figure 3. Scatter plot of biological activity in log $1 / \mathrm{Ki}$ Vs Combined dock score (CDS) 
Biological activities of the new inhibitors were predicted from the equation 2, these compounds which had better ADME properties showed comparable biological activity with that of known inhibitors. Compound $\mathrm{OHC}-1,2$ and 4 with predicted biological activity $10.579,9.987$ and 10.465 respectively were lower than known inhibitor CU 23 but comparable with CU 37, CU 29, CU 26, and CU 30.

The docking study of these known inhibitors and new molecules on the mutant variety of HIV-1 protease showed varied results. The new molecules showed better binding affinity towards the mutant variety protein. Molecules like $\mathrm{OHC} 4,5,9$ and 12 showed better docking scores $(-42.76,-36.98,-37.98$ and -38.79 respectively) than the already known inhibitors.

Since the ADME properties of the new inhibitors are better than the existing ones and they showed better binding affinity towards the protein, these molecules can be consider for further studies as hit molecules.

\section{Conclusion}

From the study of ADME properties of known inhibitors and the newly designed one which showed good bioavailability and from the docking studies performed on both wild type and mutant type protein it can be concluded that the new molecules can be considered as a potent inhibitor of HIV-1 protease. Compound like OHC-1,2, 4, 5, 9 and 12 which have better ADME properties can be taken as best hit molecules and can be considered for further studies like QSAR, synthetic studies and biological activity studies.

\section{Acknowledgements}

Schrödinger, LLC, New York is greatly acknowledged for providing the software Glide 4.0 and Qikprop 2.5. We greatly thank the Department of Chemistry, Nizam College for providing the laboratory facilities.

\section{References}

1. (a) Tomasselli A G, Howe W J, Sawyer T K, Wlodawer A and Heinrikson R L, Chimica Ogg.,1991, 6-27.(b) Huff J R, Med. Chem J, 1991, 34, 2305-2314. (c) Norbeck D W and Kempf D J, Annu. Rep, Med. Chem, 1991, 26, 141-160. (d) Debouck C, AIDS Res. Hum. Retroviruses, 1992, 8, 153-164. (e) Katz R A and Skalka A M, Annu. Rev. Biochem, 1994, 63, 133 -173.

2. (a) Wei X, Gosh S K, Taylor M E, Johnson V A, Emini E A, Deutsch P, Lifson J D, Bonhoeffer S, Nowak M A, Hahn B H, Saag M S and Shaw G M, Nature, 1995, 373 117122. (b) Ho D D, Neumann A U, Perelson A S, Chen W, Leonard J M and Markowitz M, Nature, 1995, 373,123-126. (c) Kempf D, Marsh K C, Denissen J F, McDonald E, Vasavanonda S, Flentge C A, Green B G, Fino L, Park C H, Kong X P, Wideburg N E, Saldivar A, Ruiz L, Kati W M, Sham H L, Robins T, Stewart K D, Hsu A, Plattner J J, Leonard J M and Norbeck D W, Proc. Natl. Acad. Sci. U.S.A. 1995, 92, 2484-2488.

3. Rutenber E, Fauman E B, Keenan R J, Fong S, Furth S P, Oritizde P R, Montellano D L, DeCamp, Babe L M and Craik C S, Proc. Natl. Acad. Sci, USA, 1990, 87, 6644-6648.

4. Humber D C, Cammack N, Coates J, Cobley K N, Orr D C, Storer R, Weingarten G G and Weir M P, J. Med. Chem, 1992, 35, 3080-3081.

5. Lam S P Y, Jadhav P K, Eyermann C J, Hodge C N, Ru Y, Bacheler L T, Meek J L, Otto M J, Rayner M M, Wong Y N, Chang C H, Weber P C, Sharpe T R, Jackson D A and Erickson-Viitanen S, Science, 1994, 263, 380-384.

6. Schrödinger, LLC, New York, NY, Glide, Version 4.0, 2005. 
7. QikProp, Version 2.5, Schrödinger, LLC, New York, NY, 2005.

8. Sham H L, Zhao C, Stewert K D, Betebenner D A, Lin S, Park C H, Knong X P, Rosenbrook W J, Herrin T, Madigan D, Vasavaononda S, Lyons N, Molla A, Saldivar A, Marsh K C, McDonald E, Wideburg N E, Platter J F and Norbeck D W, J. Med. Chem, 1996, 39, 392-397.

9. Yamazaki T, Hinck A P, WangY X, Nicholson L K, Torichia D A, Wingfield P, Stahl S J, Kanfman J D, Chang C H, Domaille P J and Lam P Y, Protein Sci, 1996, 5, 495-506.

10. Backbro K, Lowgran S, Osterlund K, Atepo J, Unge T, Hulten. J, Bonham N M, Schaal W, Karlen A and Halberg A, J. Med. Chem, 1997, 40, 898-902.

11. Abdel-Meguid S S, Netcalf B W, Carr T J, Demarsh P, Desjarlais R L, Fisher S, Green D W, Ivanoff L, Lambert D M and Murthy K H, Biochemistry, 994, 33, 11671-11677.

12. Chen Z, Li Y, Chen E, Hall D L, Darke P L, Culberson C, Shafer J A and Kho L C, J. Biol. Chem, 1994, 263, 344-348.

13. Thaisrivongs S, Watenpaugh K D, Howe W J, Tomich P K, Doluk L A, Chong K T, Tomich C C, Tomasselli A G, Turner S R and Strohbach J W, J. Med. Chem, 1995, 38, 3624-3637.

14. Ala P J, Huston E E, Klabe R M, McCabe D D, Duke J L, Rizzo C J, Korant B D, Deloskey R J, Lam PY, Hodge C N and Chang C H, Biochemistry, 1997, 36, 1573-1580.

15. Richard A Friesner, Jay L Banks, Robert Bm Murphy, Thomas A Halgren, Jasna J Klicic, Daniel T Mainz, Matthew P Repasky, Eric H Knoll, Mee Shelley, Jason K. Perry, David E Shaw, Perry Francis, and Peter S Shenkin J. Med. Chem, 2004, 47, 1739-174

16. Carlson H A and Masukawa K M, McCammon J A, J. Phys. Chem, 1999,103,10213-10219.

17. Carlson H A, Masukawa K M, Rubins K, Bushman F D, Jorgensen W L, Lins R D, Briggs J M, McCammon J A, J. Med. Chem. 2000, 43, 2100-2114.

18. Lam PY S, Yu Ru, Jadhav P K, Aldrich P E, DeLucca G V, Eyermann C J, Chang C H, Emmet G, Holler E R, Daneker W F, Li L, Confalone P N, Bacheler L T, Rayner M M, Klabe R M, Shum L, Winslow D L, Kornahouse D M, Jackson D A, EricksonViitanen S and C N Hodge, J. Med. Chem. 1996, 39, 3514-3525.

19. Jadhav P K, Ala P, Woerner F J, Chang C H, Garber S S, Anton E D and Bacheler L T, J. Med. Chem. 1997, 40,181 - 191.

20. Jadhav P K, Woerner F J, Lam PY S, Hodge C N, Eyermann C J, Hon-Wah Man, Daneker W F, Bacheler L T, Rayner M M, Meek J L, Erickson-Viitanen S, Jackson D A, Calabrese J C, Schadt M and Chang C H, J. Med. Chem. 1998, 41, 1446-1455.

21. Rodgers J D, Johnson B L, Wang H, Erickson-Viitanen S, Klabe R M, Bacheler L T, Cordova B C and Chang C H, Bioorg, Med. Chem, Lett.. 1998, 8, 715-720.

22. Patel M, Bacheler L T, Rayner M M, Cordova B C, Klabe R M, Erickson Viitanen S, and Sitz S P, Bioorg. Med. Chem, Lett. 1998, 8, 823-828.

23. Patel M, Kaltenbach III R F, Nugie D A, Mchugh Jr R J, Jadhav P K, Bacheler L T, Cordova B C, Klabe R M, Erickson-Viitanen S, Garber S S, Ried C and Sitz S P, Bioorg. Med. Chem, Lett. 1998, 8, 1077-1082.

24. Bhanuprakash K, Irena B, Fabio B and Stanisalav M, Biochem. Biophy. Res. Comm, 2000, 268, 384-389.

25. Thomas A H, Greenwood J R, Frye L L, Murphy and Friesner R A, WATOC - 2005, $7^{\text {th }}$ Congress of the world association of theoretically oriented chemists.

26. Lipinski C A, Lombardo F, Dominy B W and Feeney P J, Adv. Drug. Deliver. Rev. 1997, 23, 3-25.

27. Lipinski C A, Lombardo F, Dominy B W, Feeney P J, Adv. Drug. Deliver. Rev. 2001, 46, 3-26. 


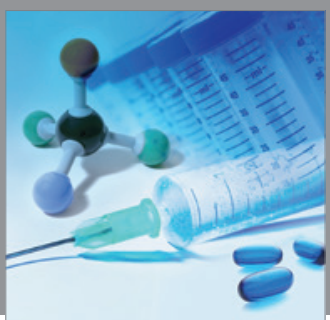

International Journal of

Medicinal Chemistry

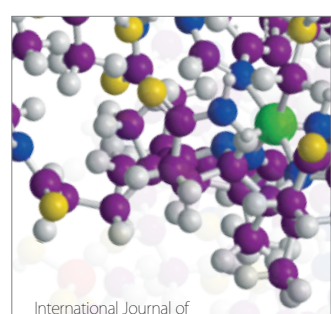

Carbohydrate Chemistry

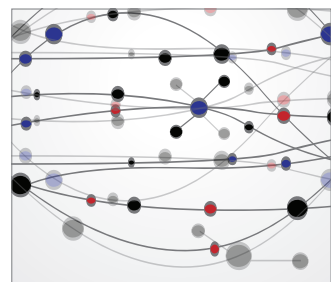

The Scientific World Journal
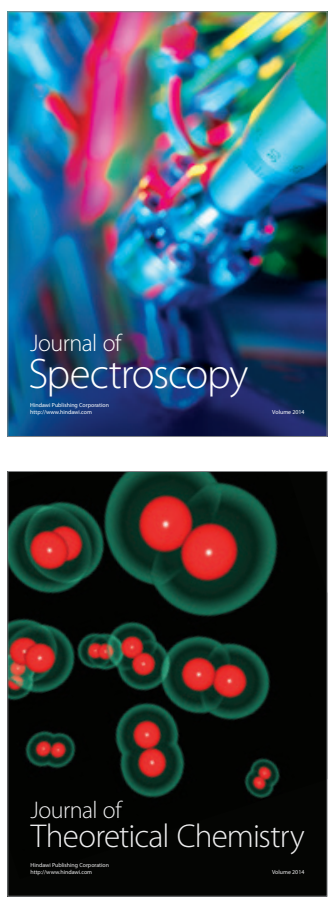
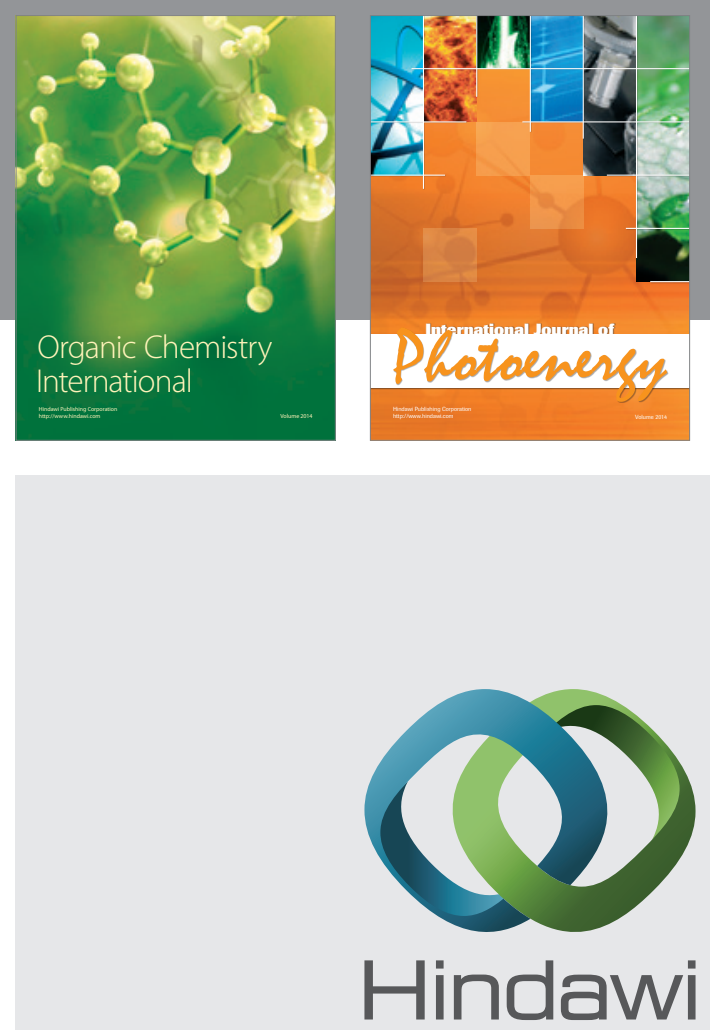

Submit your manuscripts at

http://www.hindawi.com
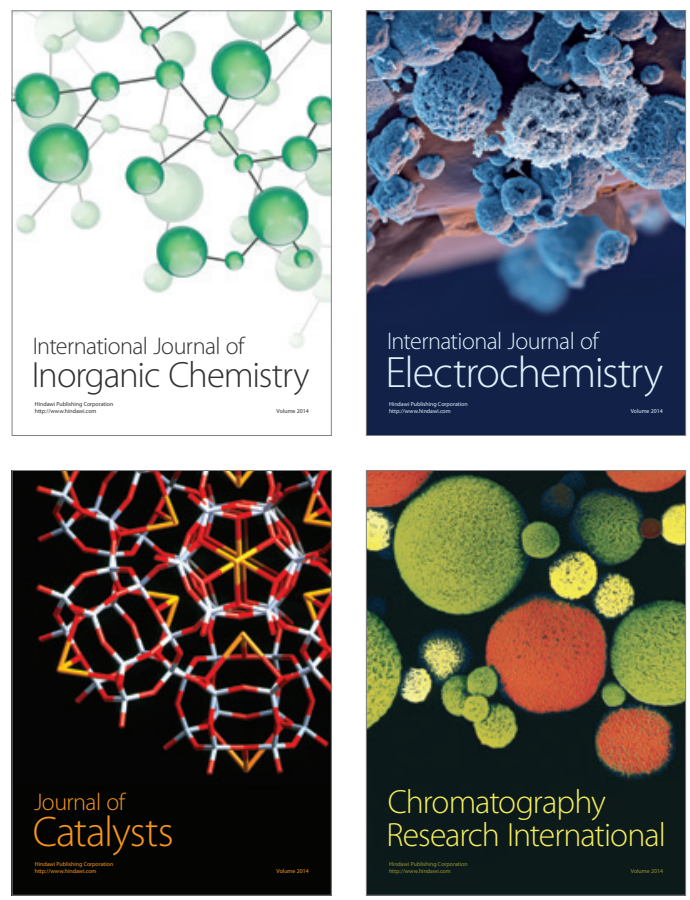
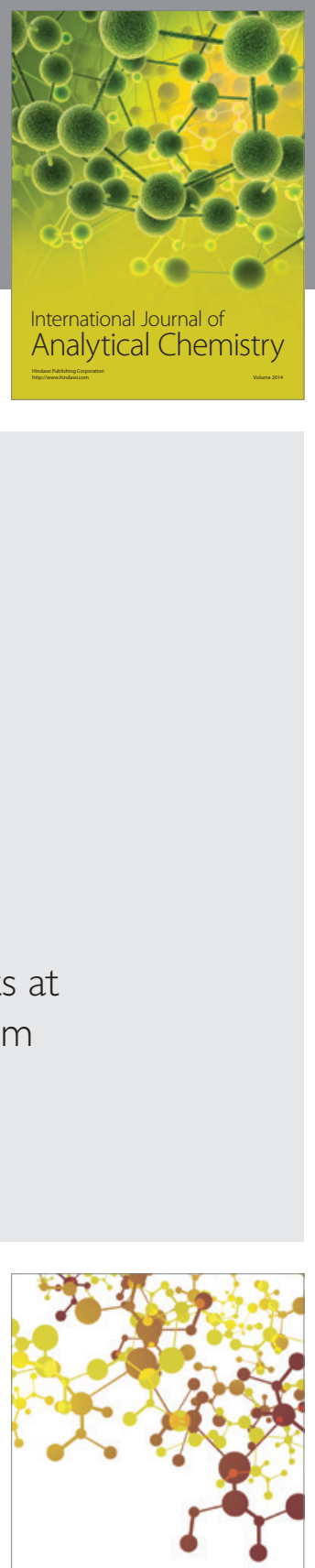

Journal of

Applied Chemistry
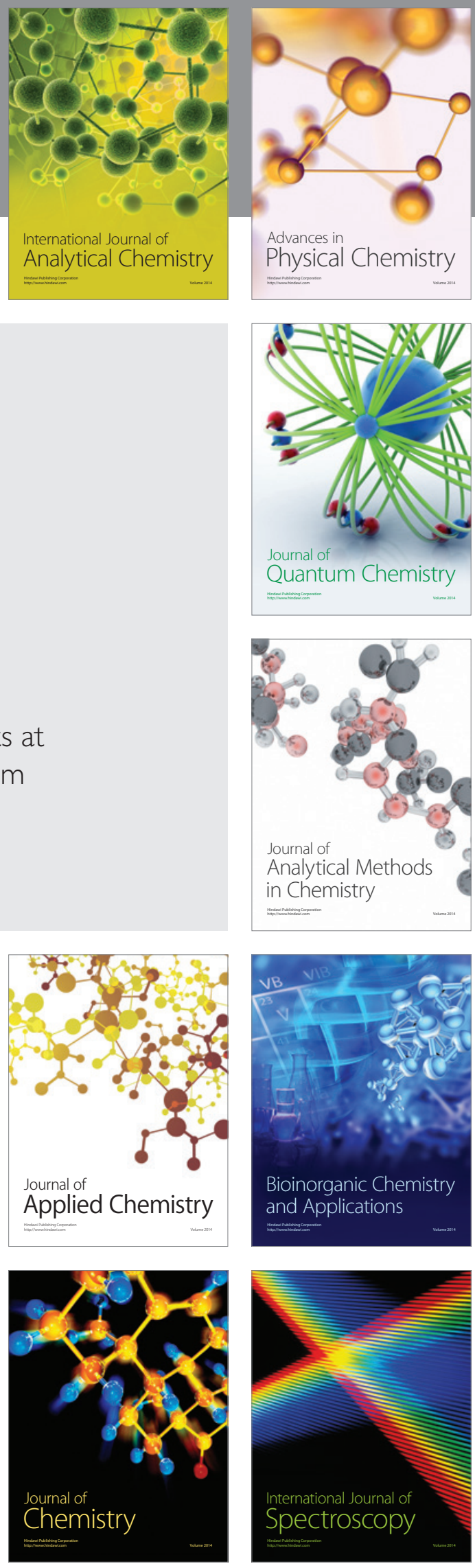